\title{
Scaling features in high energy hadron and nucleus collisions
}

\section{Zborovský*}

Nuclear Physics Institute,

Academy of Sciences of the Czech Republic,

Řež, Czech Republic

E-mail: zborovsky@ujf.cas.cz

\section{M.V. Tokarev}

Joint Institute for Nuclear Research, Dubna, Russia

E-mail: tokarevesunhe.jinr.ru

Results of analysis of experimental data on inclusive cross sections of jets, direct photons, and high- $p_{T}$ hadrons produced in $p p / \bar{p} p$ and $A A$ collisions are presented in the framework of $z-$ scaling. The data were obtained at ISR, SpppS, RHIC, and Tevatron. New properties of $z-$ presentation of the inclusive spectra are discussed. A physical interpretation of the variable $z$ and the scaling function $\psi(z)$ is given. A microscopic scenario of hadron and nucleus collisions at a constituent level in terms of momentum fractions is developed. The $z$-scaling reflects the structure of the colliding objects, interaction of their constituents, and particle formation and manifests the general properties of self-similarity, locality, and fractality. The obtained results could be used for searching for new physics phenomena in high energy hadron and nucleus collisions in soft and hard regions at RHIC, Tevatron, and LHC.

High- $p_{T}$ Physics at LHC 09

February 4-7, 2009

Prague, Czech Republic

\footnotetext{
* Speaker.
} 


\section{Introduction}

Study of particle production at high energies has revealed some regularities such as Feynman scaling [1] or P-KNO scaling [2, 3]. With the advent of new accelerators, these phenomenological laws have reached their limits of validity. Over the ISR energy range, proton-proton collisions are characterized by an increase of the multiplicity density in the central interaction region. The inclusive spectra deviate from an exponential shape at high transverse momentum approaching a more power-like behavior. Data on hadron-nucleus interactions display specific Cronin enhancement [4] when compared to proton-proton collisions. In addition to these features, particle yields measured in nucleus-nucleus collisions reveal large suppression at high $p_{T}$ relative to proton-proton data $[5,6,7,8]$. In order to distinguish between various approaches to the description of these effects, it is necessary to investigate the transverse momentum spectra of identified particles over a wide $p_{T}$ range including both soft and hard region. A careful comparison of inclusive spectra from different collision systems at different center-of-mass energies, rapidities of the inclusive particles, and associated centralities is absolutely mandatory to specify new features of heavy ion interactions.

One of the methods allowing systematic analysis of data on inclusive cross sections over a wide range of kinematical variables is based on the $z$-scaling observed in $p p$ and $\bar{p} p$ collisions [9]. These systems represent collisions of extended objects interacting in terms of their constituents. Production of particles from the constituent interactions is governed by the principles of selfsimilarity, locality, and fractality. The self-similarity of hadron production is valid both in soft and hard physics [10]. The locality and fractality are applied to the hard processes at small scales. The scaling represents independence of the scaling function $\psi(z)$ on the collision energy, type of the inclusive hadron, its production angle, and includes spectra for various selection criteria with different charged multiplicities [11]. The general principles can be applied to the nucleus-nucleus interactions as well. We demonstrate that the charged hadron and pion spectra in $A A$ collisions for different centrality classes characterized by different multiplicity densities $d N_{\mathrm{ch}} / d \eta$ exhibit similar scaling behavior as in $p p$ interactions. This holds in a wide range of the transverse momentum $p_{T}$ and the collision energy $\sqrt{s}$. The transverse momentum distributions for $A A$ systems depend on the collision centrality and differ from the scaling behavior for $p p$ system at high $p_{T}$. In the case of charged hadrons and pions, a multiplicity dependence of the scaling parameter $\varepsilon_{A A}$ interpreted as a fragmentation dimension allows us to restore the identical shape of the scaling function $\psi(z)$ in $A A$ collisions for all centralities. This shape turns out to coincide with the $z$-scaling in $p p$ interactions. The multiplicity dependence of $\varepsilon_{A A}$ is connected with an increase of the energy losses of high- $p_{T}$ particles in the central collisions of heavy nuclei.

\section{2. $z$-Scaling}

The concept of $z$-scaling is based on some simple ideas. It is assumed that the collision of extended objects like hadrons and nuclei at sufficiently high energies is considered as an ensemble of individual interactions of their constituents. The constituents are partons in the parton model or quarks and gluons in the theory of QCD. A single interaction of the constituents is depicted in Fig.1. The structures of the colliding objects are characterized by parameters $\delta_{1}$ and $\delta_{2}$. The constituents of the incoming objects with masses $M_{1}, M_{2}$ and momenta $P_{1}, P_{2}$ carry their fractions $x_{1}, x_{2}$. The 


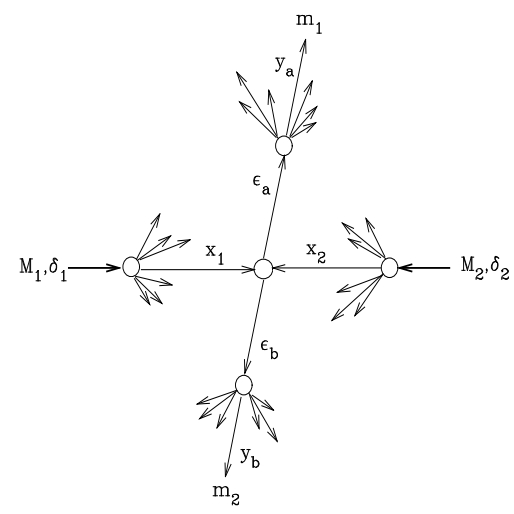

Figure 1: Diagram of the constituent subprocess.

inclusive particle carries the momentum fraction $y_{a}$ of the scattered constituent with a fragmentation characterized by a parameter $\varepsilon_{a}$. A fragmentation of the recoil constituent is described by the parameter $\varepsilon_{b}$ and the momentum fraction $y_{b}$. Multiple interactions of constituents are considered to be similar. This property reflects self-similarity of hadronic interactions at a constituent level.

\subsection{Momentum fractions $x_{1}, x_{2}, y_{a}$, and $y_{b}$}

The idea of the $z$-scaling relies on the assumption [12] that gross features of an inclusive particle distribution of the reaction

$$
M_{1}+M_{2} \rightarrow m_{1}+X
$$

can be described at high energies in terms of the kinematical characteristics of the corresponding constituent subprocess. We consider the subprocess

$$
\left(x_{1} M_{1}\right)+\left(x_{2} M_{2}\right) \rightarrow\left(m_{1} / y_{a}\right)+\left(x_{1} M_{1}+x_{2} M_{2}+m_{2} / y_{b}\right)
$$

written in the symbolic form to be a binary collision of the constituents $\left(x_{1} M_{1}\right)$ and $\left(x_{2} M_{2}\right)$ resulting in the scattered $\left(m_{1} / y_{a}\right)$ and recoil $\left(x_{1} M_{1}+x_{2} M_{2}+m_{2} / y_{b}\right)$ objects in the final state. The registered particle with the 4-momentum $p$ and mass $m_{1}$ carries the fraction $y_{a}$ of the 4-momentum of the object produced in the binary subprocess into the observed direction. Its counterpart $\left(m_{2}\right)$, moving in the opposite direction, carries the 4-momentum fraction $y_{b}$ of the produced recoil. The associate production of $\left(m_{2}\right)$ ensures conservation of the additive quantum numbers. The produced secondary objects transform into real particles after the constituent collisions.

The momentum conservation law of the constituent subprocess is connected with a recoil mass as follows

$$
\left(x_{1} P_{1}+x_{2} P_{2}-p / y_{a}\right)^{2}=M_{X}^{2} .
$$

We write the recoil mass in the form

$$
M_{X}=x_{1} M_{1}+x_{2} M_{2}+m_{2} / y_{b}
$$


The quantity $M_{X}$ is proportional to the fractions $x_{1}$ and $x_{2}$ of the masses $M_{1}$ and $M_{2}$. Dominant contribution to the recoil mass is given by the term $m_{2} / y_{b}$. The value of $M_{X}$ is calculated by (2.4) with the values of $x_{1}, x_{2}$, and $y_{b}$ determined by a method described bellow. Equation (2.3) is an expression of the locality of the hadron interaction at a constituent level. Expressions (2.3) and (2.4) represent a constraint on the momentum fractions $x_{1}, x_{2}, y_{a}$, and $y_{b}$ which determine a subprocess (2.2).

A specific situation is the kinematical limit which can be achieved kinematically at any energy. In this case the whole reaction (2.1) degenerates to a single subprocess (2.2) and all momentum fractions become unity, $x_{1}=x_{2}=y_{a}=y_{b}=1$. The kinematical limit corresponds to the binary configuration in which the recoil object forms a cluster with the invariant mass $M_{X}=M_{1}+M_{2}+m_{2}$. To be more specific, let us take for example the inclusive production of $K^{-}$-mesons in proton-proton collisions. The main channel of $K^{-}$production is usually considered with simultaneous formation of $K^{+}$meson. Here the kinematical limit corresponds to the exclusive reaction $p+p \rightarrow K^{-}+p+$ $p+K^{+}$with $K^{-}$-meson produced opposite to a cluster with the invariant mass which equals to the sum of the masses of three particles $p, p$, and $K^{+}, M_{X}=M(p)+M(p)+m\left(K^{+}\right)$. This condition reflects validity of internal conservation laws (for isospin, baryon number, strangeness,...).

Structure of the colliding objects and fragmentation of the systems formed in the scattered and recoil directions are characterized by the parameters $\delta_{1}, \delta_{2}$ and $\varepsilon_{a}, \varepsilon_{b}$, respectively. We connect the structural parameters with the corresponding momentum fractions by the function

$$
\Omega\left(x_{1}, x_{2}, y_{a}, y_{b}\right)=\left(1-x_{1}\right)^{\delta_{1}}\left(1-x_{2}\right)^{\delta_{2}}\left(1-y_{a}\right)^{\varepsilon_{a}}\left(1-y_{b}\right)^{\varepsilon_{b}} .
$$

Physical interpretation of $\Omega$ is given by its proportionality to relative number of all such constituent configurations in the reaction (2.1) which contain the configuration defined by the fractions $x_{1}, x_{2}, y_{a}$, and $y_{b}$. The function $\Omega$ plays the role of a relative volume which occupy these configurations in the space of the momentum fractions. It was found that the structural parameters $\delta_{1}$ and $\delta_{2}$ have constant values at high energies. This holds for $\varepsilon_{a}$ and $\varepsilon_{b}$ in $p p$ collisions as well. The parameters are interpreted as fractal dimensions in the corresponding space of the momentum fractions. For proton-proton collisions we set $\delta_{1}=\delta_{2} \equiv \delta$. In the case of nucleus-nucleus collisions there are relations $\delta_{1}=A_{1} \delta$ and $\delta_{2}=A_{2} \delta$, where $A_{1}, A_{2}$ are atomic numbers [13]. We assume that the fragmentation of the objects moving in the scattered and recoil directions can be described by the same parameter $\varepsilon_{a}=\varepsilon_{b} \equiv \varepsilon_{F}$ which depends on the type $(F)$ of the inclusive particle. For given values of $\delta$ and $\varepsilon_{F}$, we determine the fractions $x_{1}, x_{2}, y_{a}$, and $y_{b}$ in a way to maximize the function $\Omega\left(x_{1}, x_{2}, y_{a}, y_{b}\right)$, simultaneously fulfilling condition (2.3) where $M_{X}$ is given by equation (2.4). The momentum fractions $x_{1}$ and $x_{2}$ obtained in this way can be decomposed as follows

$$
x_{1}=\lambda_{1}+\chi_{1}, \quad x_{2}=\lambda_{2}+\chi_{2},
$$

where $\lambda_{1,2}=\lambda_{1,2}\left(y_{a}, y_{b}\right)$ and $\chi_{1,2}=\chi_{1,2}\left(y_{a}, y_{b}\right)$ are specific functions of $y_{a}$ and $y_{b}$ [9]. Using the decomposition, the subprocess (2.2) can be rewritten into a symbolic form

$$
x_{1}+x_{2} \rightarrow\left(\lambda_{1}+\lambda_{2}\right)+\left(\chi_{1}+\chi_{2}\right) .
$$

This relation means that the $\lambda$-parts of the interacting constituents contribute to the production of the inclusive particle, while the $\chi$-parts are responsible for the creation of its recoil. 
Since the momentum fractions are determined by means of the maximization of the expression (2.5), they implicitly depend on $\delta$ and $\varepsilon_{F}$. The parameter $\varepsilon_{F}$ takes effectively into account also prompt resonances out of which the inclusive particle of a given type may be created. At fixed mass parameter $m_{2}$, larger values of $\varepsilon_{F}$ correspond to smaller $y_{a}$ and $y_{b}$, which in turn give larger ratios $m_{2} / y_{b}$ and $m_{1} / y_{a}$. In our phenomenological approach this means that production of the inclusive particle $\left(m_{1}\right)$ and its counterpart $\left(m_{2}\right)$ is a result of fragmentation from larger masses which mimic in a sense processes with prompt resonances. Values of these parameters are determined in accordance with self-similarity requirements and experiment. In particular, this gives the restriction $m_{2}=m_{1}$.

\subsection{Scaling variable $z$ and scaling function $\psi(z)$}

The self-similarity of hadron interactions reflects a property that hadron constituents and their interactions are similar. This is connected with dropping of certain dimensional quantities out of the description of physical phenomena. The self-similar solutions are constructed in terms of the self-similarity parameters. We search for a solution

$$
\psi(z)=\frac{1}{N \sigma_{\text {inel }}} \frac{d \sigma}{d z}
$$

depending on a single self-similarity variable $z$. Here $\sigma_{\text {inel }}$ is an inelastic cross section of the reaction (2.1) and $N$ is an average particle multiplicity. The variable $z$ is defined as follows

$$
z=z_{0} \Omega^{-1}
$$

where

$$
z_{0}=\frac{\sqrt{s_{\perp}}}{\left(d N_{c h} /\left.d \eta\right|_{0}\right)^{c} m}
$$

and $\Omega$ given by (2.5). For a given reaction (2.1), $z$ is proportional to the transverse kinetic energy $\sqrt{s_{\perp}}$ of the constituent subprocess (2.2) consumed on the production of the inclusive particle $\left(m_{1}\right)$ and its counterpart $\left(m_{2}\right)$. The energy $\sqrt{s_{\perp}}$ is determined by the formula

$$
\sqrt{s_{\perp}}=T_{a}+T_{b},
$$

where

$$
T_{a}=y_{a}\left(\sqrt{s_{\lambda}}-M_{1} \lambda_{1}-M_{2} \lambda_{2}\right)-m_{1}, \quad T_{b}=y_{b}\left(\sqrt{s_{\chi}}-M_{1} \chi_{1}-M_{2} \chi_{2}\right)-m_{2} .
$$

The terms

$$
\sqrt{s_{\lambda}}=\left[\left(\lambda_{1} P_{1}+\lambda_{2} P_{2}\right)^{2}\right]^{1 / 2}, \quad \sqrt{s_{\chi}}=\left[\left(\chi_{1} P_{1}+\chi_{2} P_{2}\right)^{2}\right]^{1 / 2}
$$

represent the energy for production of the secondary objects moving in the scattered and recoil direction, respectively. The quantity $d N_{c h} /\left.d \eta\right|_{0}$ is the corresponding multiplicity density of charged particles in the central region of the reaction (2.1) at pseudorapidity $\eta=0$. The multiplicity density in the central interaction region is related to a state of the produced medium. The parameter $c$ characterizes properties of this medium [9]. It is determined from multiplicity dependence of inclusive spectra $[9,10,11]$. The mass constant $m$ is an arbitrary one and we fix it at the value of a nucleon mass. 
The scaling function $\psi(z)$ is expressed in terms of the experimentally measured inclusive cross section $E d^{3} \sigma / d p^{3}$, the multiplicity density $d N / d \eta$ at pseudorapidity $\eta$, and $\sigma_{\text {inel }}$. Exploiting the definition (2.8) one can obtain the expression [9]

$$
\psi(z)=-\frac{\pi s}{(d N / d \eta) \sigma_{\text {inel }}} J^{-1} E \frac{d^{3} \sigma}{d p^{3}},
$$

where $s$ is the square of the center-of-mass energy and $J$ is the corresponding Jacobian. The multiplicity density $d N / d \eta$ in the expression (2.14) concerns particular hadrons species. It depends on the center-of-mass energy, on various multiplicity selection criteria, and also on the production angles at which the inclusive spectra were measured. The procedure of obtaining the corresponding values of $d N / d \eta$ from the $p_{T}$ spectra is described in Ref. [9]. The function $\psi(z)$ is normalized as follows

$$
\int_{0}^{\infty} \psi(z) d z=1
$$

The above relation allows us to interpret the function $\psi(z)$ as a probability density to produce an inclusive particle with the corresponding value of the variable $z$.

\section{Self-similarity in $p p \boldsymbol{\&} \bar{p} p$ collisions}

\subsection{Hadrons}

The self-similarity of hadron production in $p p(\bar{p} p)$ collisions at high energies is manifested by the energy, angular, and multiplicity independence of the scaling function $\psi(z)$ for different types of the inclusive hadrons. This was shown in Ref. [9] for charged and identified $(\pi, K, \bar{p})$ hadrons produced in $p p$ collisions over a wide range of the center-of-mass energy $\sqrt{s}$, detection angle $\theta_{c m s}$, and multiplicity density $d N / d \eta$. The energy, angular, and multiplicity independence of $\psi(z)$ was obtained for the constant values of $\delta=0.5$ and $c=0.25$. The parameter $\varepsilon_{F}$ was found to increase with the hadron mass. The values of $d N_{c h} /\left.d \eta\right|_{0}$ were taken for the inelastic collisions. The parameter $m_{2}$ was determined from the corresponding exclusive reactions at the kinematical limit $\left(x_{1}=x_{2}=y_{a}=y_{b}=1\right)$. This gives $m_{2}=m\left(\pi^{+}\right), m_{2}=m\left(K^{+}\right)$, and $m_{2}=m(p)$, for the inclusive production of $\pi^{-}, K^{-}$, and antiprotons, respectively. The same relation, $m_{2}=m_{1}$, was used for all types of inclusive hadrons. For comparison of the $z$-presentation of the spectra for different hadron species we exploit the transformation

$$
z \rightarrow \alpha_{F} z, \quad \psi \rightarrow \alpha_{F}^{-1} \psi
$$

The scale transformation allows to collapse the scaling functions of different hadrons to a single curve. The value $\alpha_{\pi}=1$ for pions was chosen as a reference. Figure 2 shows $z$-presentation of the spectra of negative pions, kaons, antiprotons, and $\Lambda^{\prime} s$ produced in $p p$ collisions over the range $\sqrt{s}=19-200 \mathrm{GeV}$ and $\theta_{c m s}=3^{0}-90^{0}$. The symbols represent data on differential cross sections measured in the central $[14,15]$ and fragmentation [16] regions, as well as spectra [17] measured up to very small transverse momenta. The solid lines and single data sets are shifted by multiplicative factors for clarity. The indicated values of the parameters $\varepsilon_{F}$ and $\alpha_{F}$ are consistent with the energy, angular, multiplicity, and flavor independence of the $z$-presentation of spectra for different hadrons. The parameters are independent of kinematical variables $\left(\sqrt{s}, p_{T}\right.$, and $\left.\theta_{c m s}\right)$. The distributions of 


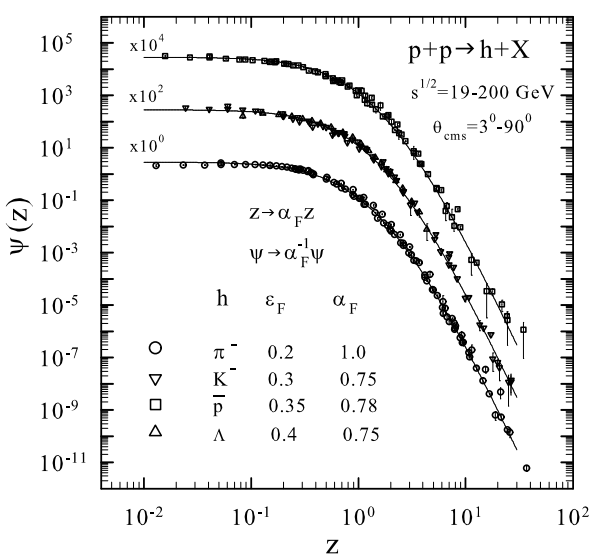

Figure 2: The energy, angular, and flavor independence of $z$-scaling in $p p$ collisions. The solid lines represent the same curve. Data are taken from Refs. [14, 15, 16, 17].

different hadrons are sufficiently well described by a single curve over a wide $z$-range. The scaling function reveals a saturation for $z<0.1$. Similar results for other hadrons $\left(\rho, \omega, \phi, K^{*}, \Xi\right)$ produced

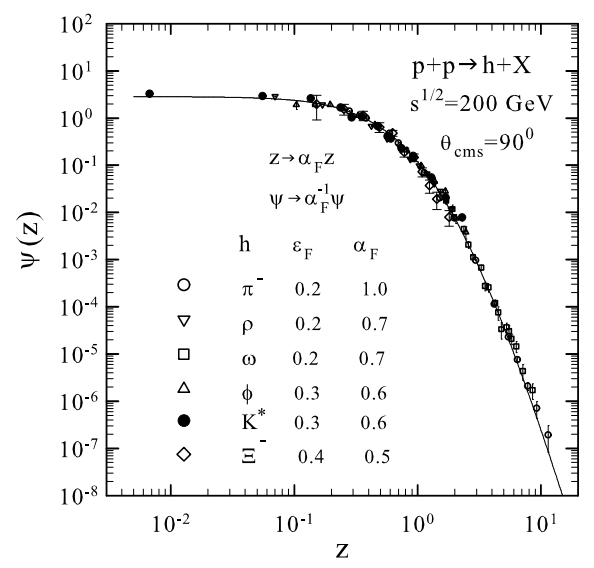

a)

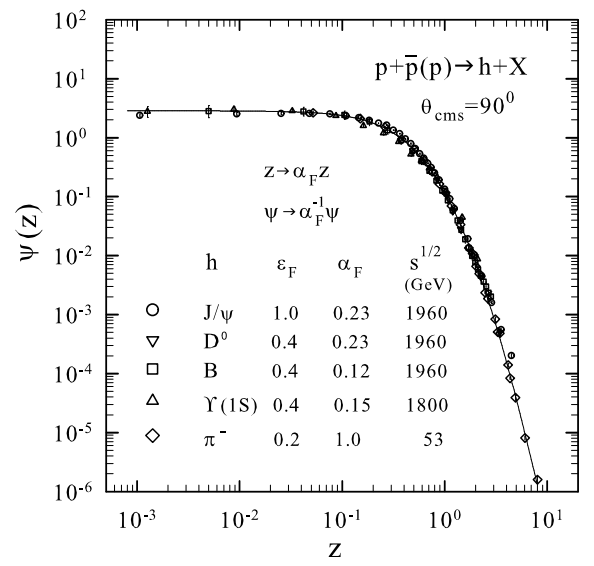

b)

Figure 3: The flavor independence of $z$-scaling. The spectra of $\pi^{-}, \rho, \omega, \phi, K^{*}, \Xi$ hadrons $[15,18]$ produced in $p p$ collisions at RHIC (a) and $J / \psi, D^{0}, B, \Upsilon$, mesons [19] produced in $\bar{p} p$ collisions at Tevatron (b) in $z$-presentation. The solid lines are the same curves as in Fig. 2.

in $p p$ collisions at $\sqrt{s}=200 \mathrm{GeV}$ and $\theta_{c m s}=90^{\circ}$ are shown in Fig.3(a). The data are compared with the pion distributions obtained at RHIC. The shape of $\psi(z)$ is described by the same curve as depicted in Fig. 2. Figure 3(b) shows the spectra of $J / \psi, D^{0}, B$, and $\Upsilon$ mesons measured at the Tevatron energies $\sqrt{s}=1800$ and $1960 \mathrm{GeV}$ in $z$-presentation. The scaling function reveals a saturation in the range $z=0.001-0.1$ and is the same as for hadrons with light flavors.

\subsection{Direct photons}

The D0 Collaboration published the data [20] on inclusive cross sections of direct photons produced in $\bar{p} p$ collisions at $\sqrt{s}=1960 \mathrm{GeV}$. The data cover the momentum $p_{T}=30-250 \mathrm{GeV} / \mathrm{c}$ 


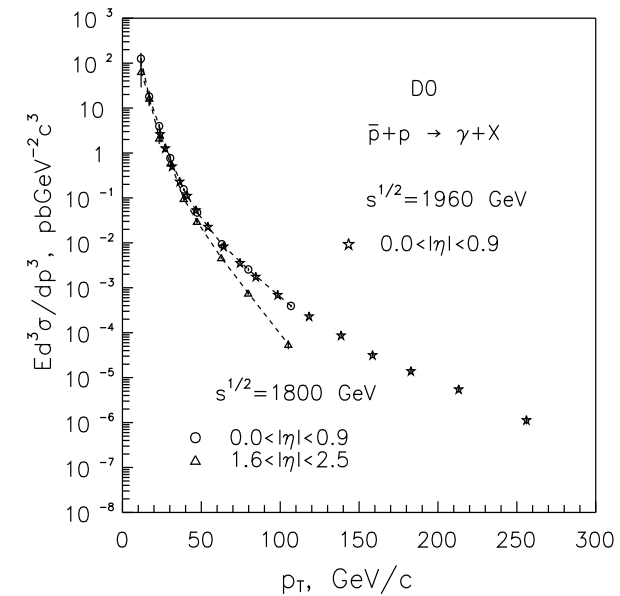

a)

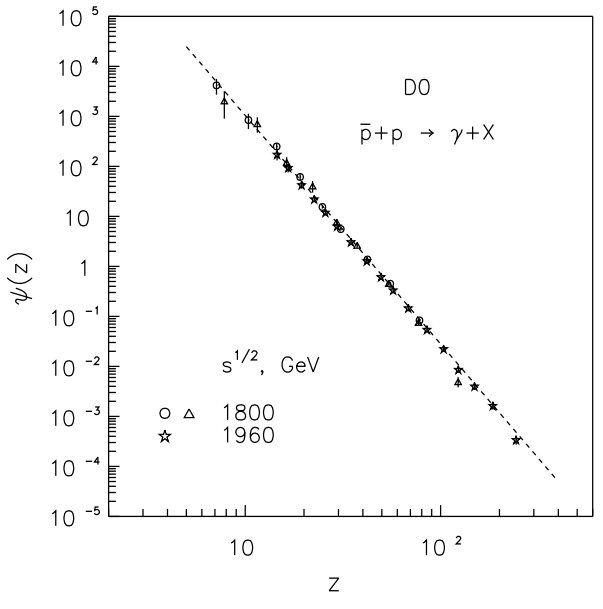

b)

Figure 4: Spectra of direct photons produced in $\bar{p} p$ collisions in (a) $p_{T^{-}}$and (b) $z$-presentations. Experimental data obtained by D0 Collaboration in Run I and II are taken from Ref. [20].

and pseudorapidity $|\eta|<0.9$ range. These data together with the data obtained by D0 Collaboration in Run I are presented in Fig. 4(a). A strong angular dependence of the cross section is observed. It increases with $p_{T}$ and reaches about one order of magnitude at $p_{T}=100 \mathrm{GeV} / \mathrm{c}$. The $z$-presentation of the same data is shown in Fig. 4(b). In this case $\varepsilon=0$ was used. One can see that experimental data confirm the features (energy and angular independence) of the $z$-scaling for direct photon production in $\bar{p} p$ and $p p$ collisions established in Ref. [21]. The power law, $\psi(z) \sim z^{-\beta}$, is observed over a wide range of $z$. The independence of the slope parameter $\beta$ on kinematical variables $\left(\sqrt{s}, p_{T}\right.$, and $\left.\eta\right)$ is considered as an evidence of self-similarity and fractality of photon production over a wide scale range.

\subsection{Jets}

The experimental data on inclusive cross sections of jet production at different collision energies obtained by the D0 [22, 23] and CDF [24, 25] Collaborations at Tevatron allow us to study properties of the function $\psi(z)$. Figure 5(a) shows $z$-presentation of the jet spectra. All data demonstrate the energy independence of the scaling function. A power behavior of $\psi(z)$ is valid over a wide range of $z$. The scaling function changes more than twelve orders of magnitude. The obtained results manifest self-similarity in jet production.

The angular dependence of jet production in $\bar{p} p$ collisions at $\sqrt{s}=1960 \mathrm{GeV}$ was investigated by the CDF Collaboration. The experimental data [26] cover the pseudorapidity range $|\eta|<2.1$. The highest transverse energy carried by one jet is about $600 \mathrm{GeV}$. The transverse momentum spectra of these data are shown on Fig. 6(a). They show a strong dependence on the pseudorapidity of the produced jet. The $z$-presentation of the same data is presented in Fig. 6(b). It demonstrates the angular independence and the power behavior of $\psi(z)$ over the range $z \simeq 100-1000$.

Figure 7 shows inclusive spectra [27] of jet production in $\bar{p} p$ collisions at the center-of-mass energy $\sqrt{s}=1960 \mathrm{GeV}$, over the momentum $p_{T}=50-600 \mathrm{GeV} / \mathrm{c}$ and pseudorapidity $|\eta|<2.4$ ranges in $p_{T}$ - and $z$-presentations. The data collected by the D0 Collaboration correspond to the 


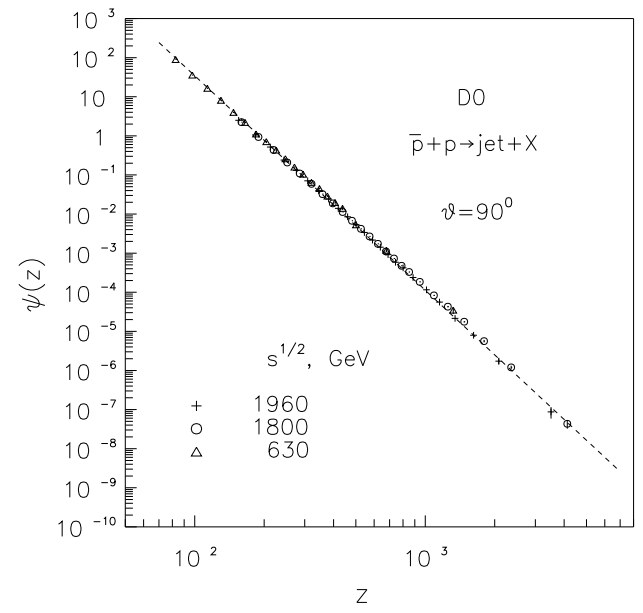

a)

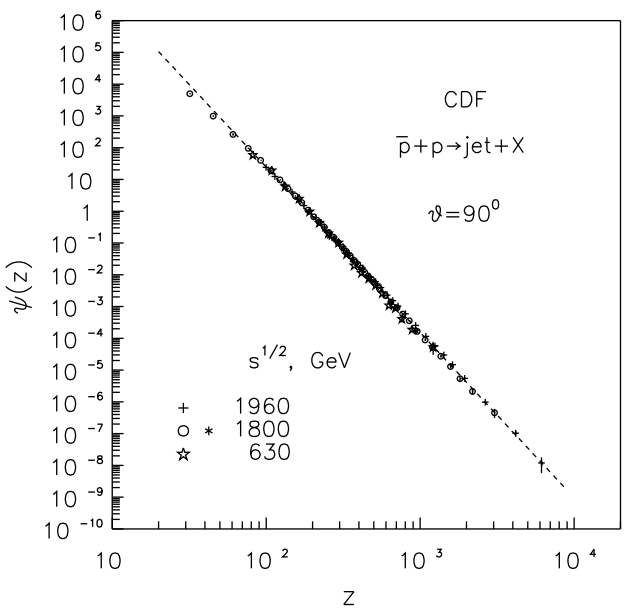

b)

Figure 5: Inclusive spectra of jet production in $\bar{p} p$ collisions at $\sqrt{s}=630,1800$, and $1960 \mathrm{GeV}$ and $\theta_{\text {cms }} \simeq$ $90^{\circ}$ measured by the D0 [22, 23] and CDF [24, 25] Collaborations are shown in z-presentation.

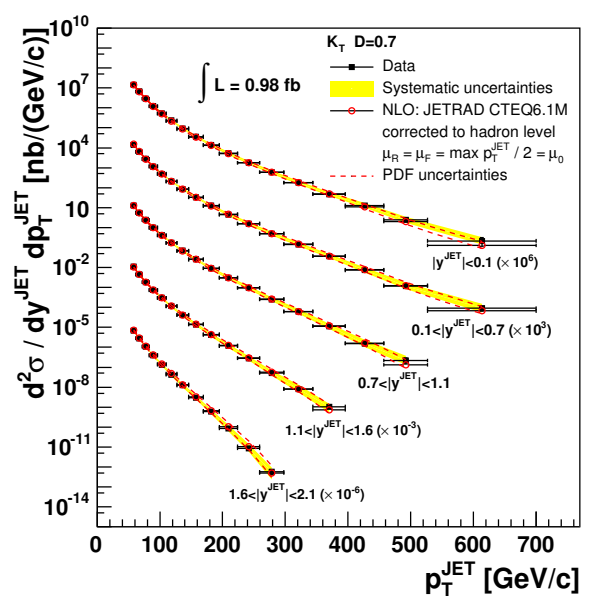

a)

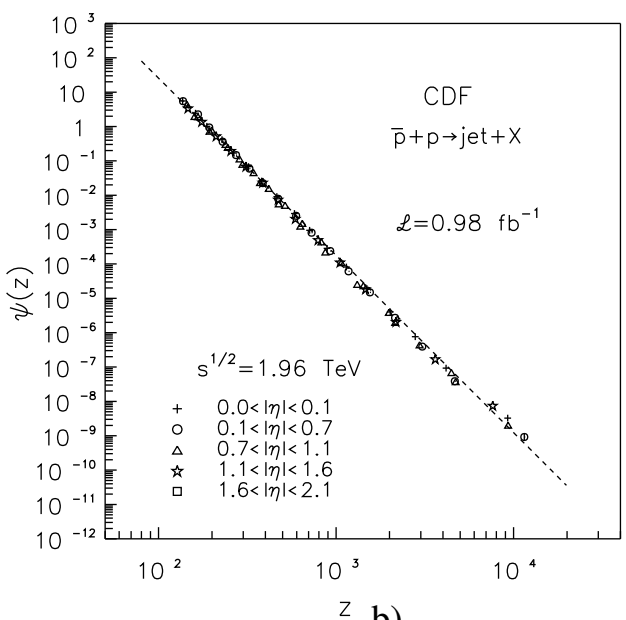

b)

Figure 6: Inclusive spectra of jet production in $\bar{p} p$ collisions at $\sqrt{s}=1960 \mathrm{GeV}$ and different pseudorapidity intervals in (a) $p_{T}$ - and (b) $z$-presentations. Experimental data obtained by the CDF Collaboration are taken from Ref. [26].

integrated luminosity of $0.7 \mathrm{fb}^{-1}$. As seen from Fig. 7(a), the spectra measured for different pseudorapidity intervals demonstrate a strong angular dependence. It is enhanced with increasing of the transverse momentum. The jet yields decrease in the measured momentum range of $p_{T}$ by more than ten orders of magnitude. The theoretical calculations of the jet spectra in the NLO QCD (see Ref. [27] and references therein) shown by the solid lines are in a good agreement with the data. Figure 7(b) demonstrates the angular independence of the corresponding scaling function $\psi(z)$ over a wide kinematical range. The function is described by the power law, $\psi(z) \sim z^{-\beta}$, with a constant value of the slope parameter $\beta=5.48 \pm 0.02$. 


\section{Scaling features of charged hadron spectra in $A A$ collisions}

The measurements of particle spectra at RHIC led to the discovery of a substantial suppression of hadron yields in nucleus-nucleus collisions relative to proton-proton data. The suppression is observed in the region of high transverse momenta, typically more than few GeV/c. It is usually connected with the energy radiations of the outgoing high- $p_{T}$ partons propagating through dense matter formed in the central collisions of heavy nuclei. The energy losses in the dense medium are substantially larger than in the vacuum. One of the measurable quantities characterizing the medium is the multiplicity density of the produced particles. We present some ideas how to quantify the energy losses exploiting a specific connection between the suppression of the spectra and the corresponding multiplicity density of particles produced in nuclear collisions. The ideas are motivated by the assumption of self-similarity of hadron interactions at a constituent level both in $p p$ and $A A$ collisions.

We have analyzed data $[5,8,28,29]$ on inclusive distributions of charged hadrons measured in $A u A u$ and $C u C u$ collisions at RHIC. Figure 8(a) shows a comparison of the transverse momentum spectra from the peripheral $A u A u$ collisions at the energies $\sqrt{s_{N N}}=63,130$, and $200 \mathrm{GeV}$ with the corresponding data from $p p$ interactions. All spectra were measured in the central pseudorapidity region.

The same data are depicted in Fig. 8(b) in $z$-presentation. The parameter $\varepsilon=0.2$ was fixed at the value obtained from the scaling analysis of the charged particles in $p p$ collisions. The fractal dimension $\delta_{A}$ of the colliding nuclei was determined in accordance with the additive property $\delta_{A}=A \delta$ for $p A$ interactions [13] with the same value of $\delta=0.5$ as for $p p$ data. The total multiplicity densities $d N_{\mathrm{ch}} /\left.d \eta\right|_{0}$ of charged particles produced in $A A$-collisions have been used in formula (2.10) for nuclear interactions. In the case of $p p$ interactions, the corresponding multiplicity densities $d N_{\mathrm{ch}} /\left.d \eta\right|_{0}$ for the non-single-diffractive $p p$ events were used in (2.10) for comparative reasons.

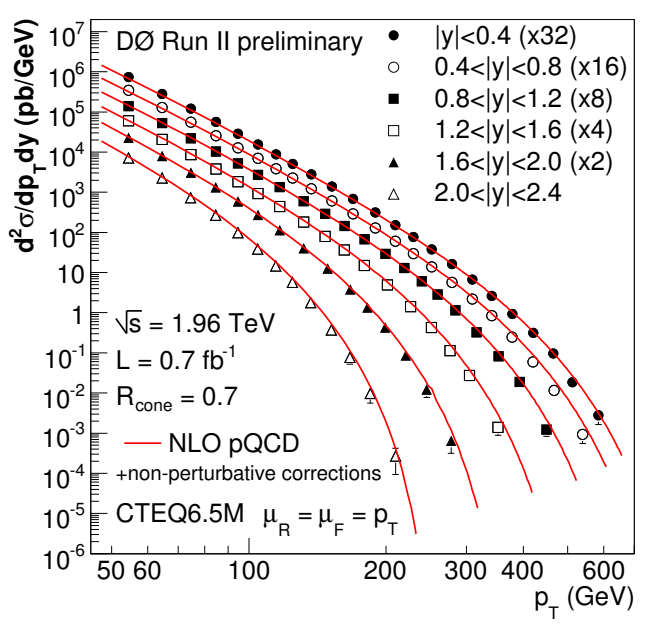

a)

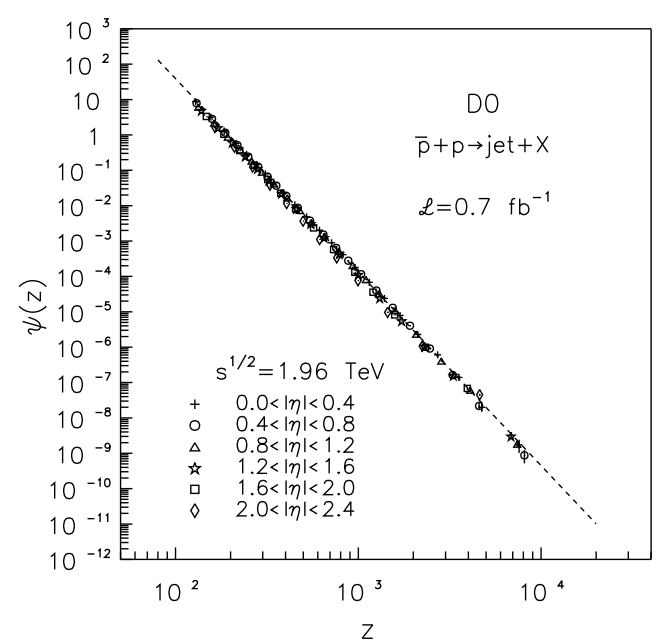

b)

Figure 7: Inclusive spectra of jet production in $\bar{p} p$ collisions at $\sqrt{s}=1960 \mathrm{GeV}$ and different pseudorapidity intervals in (a) $p_{T^{-}}$and (b) $z$-presentations. Experimental data obtained by the Do Collaboration are taken from Ref. [27]. 


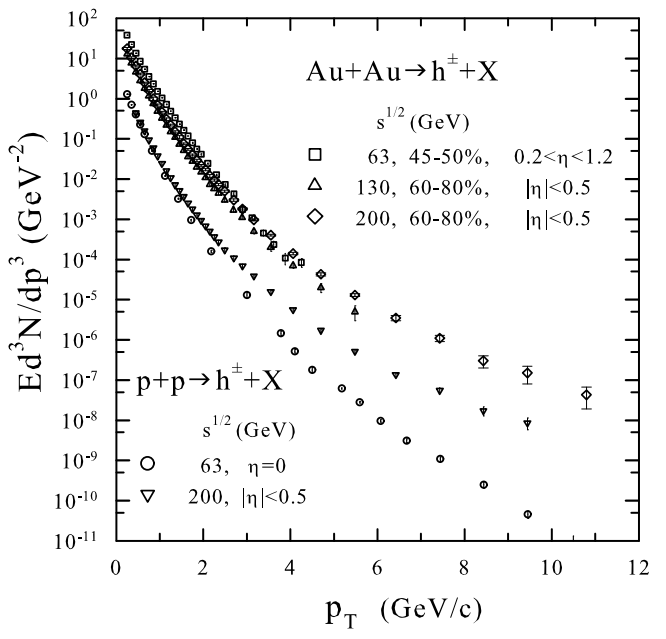

a)

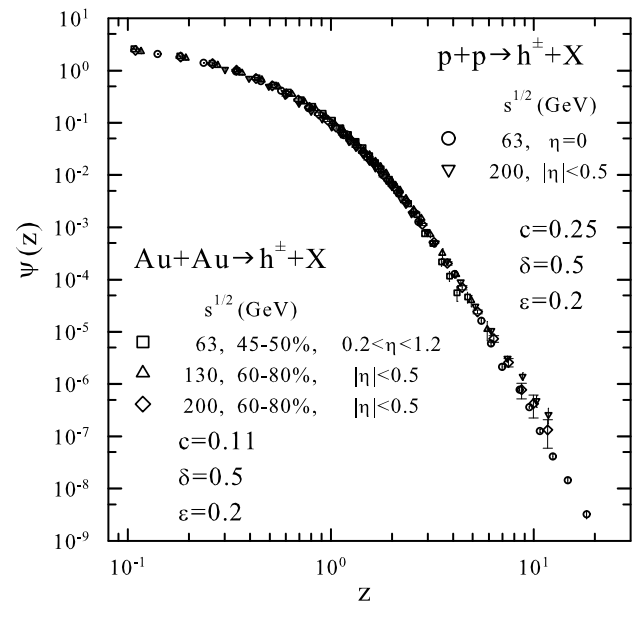

b)

Figure 8: Inclusive spectra of charged hadrons produced in $p p$ and peripheral $A u A u$ collisions at different energies $\sqrt{s}_{N N}=63-200 \mathrm{GeV}$ in (a) $p_{T}$ - and (b) z-presentations. Experimental data are taken from Refs. $[5,8,28,30,31]$.

The energy independence of $z$-presentation of the spectra in the peripheral $A u A u$ collisions was obtained for $c_{\mathrm{AuAu}}=0.11$. As seen from Fig. 8(b), the shape of $\psi(z)$ in the peripheral $A u A u$ collisions coincides with good accuracy with the $z$-scaling in $p p$ interactions. The result indicates that form of the charged hadron spectra in peripheral nuclear collisions is unsensitive to modifications of the production mechanism by nuclear medium when compared with $p p$ system. The influence of nuclei is included here with a drop-off in the parameter $c$ interpreted as a "specific heat" [32].

We have calculated $\psi(z)$ for different centralities characterized by different multiplicity densities $d N_{\mathrm{ch}} /\left.d \eta\right|_{0}$. Results of the calculations for the STAR data [5] on charged hadrons produced in $A u A u$ collisions at $\sqrt{s_{N N}}=200 \mathrm{GeV}$ and $|\eta|<0.5$ are shown in Fig. 9(a). One can see clear suppression of the $z$ presentation of the spectra with the increasing centrality in $A u A u$ collisions relative to the $z$ scaling observed in $p p$ interactions (line). As shown in Fig. 9(b), the same scaling behavior as for $p p$ collisions can be restored for $A u A u$ interactions for all centralities. This can be achieved by the parameter $\varepsilon$ allowing it to be a function of the multiplicity density $d N_{\mathrm{ch}} / d \eta$. For that purpose we have used the simple linear dependence

$$
\varepsilon_{A A}=\varepsilon_{0}\left(d N_{\mathrm{ch}} / d \eta\right)+\varepsilon_{p p}
$$

For $A u A u$ collisions at $\sqrt{s_{N N}}=200 \mathrm{GeV}$ we obtained $\varepsilon_{0}=0.0028$.

Similar results are valid for $\mathrm{CuCu}$ system. The $z$-presentation of the charged hadron spectra with the multiplicity independent parameter $\varepsilon_{\mathrm{CuCu}}=\varepsilon_{p p}=0.2$ is shown in Fig. 10(a). The points correspond to the data [29] on hadron yields for various centralities measured by the PHOBOS Collaboration at $\sqrt{s_{N N}}=200 \mathrm{GeV}$ in the pseudorapidity range $0.2<\eta<1.4$. The parameter $c$ ("specific heat") was determined from the energy independence of $z$-presentation of the spectra in the peripheral $\mathrm{CuCu}$ collisions. The obtained value $c_{\mathrm{CuCu}}=0.14$ lies between the corresponding values for $A u A u$ and $p p$ systems. Though uncertainty of its determination from the available data 


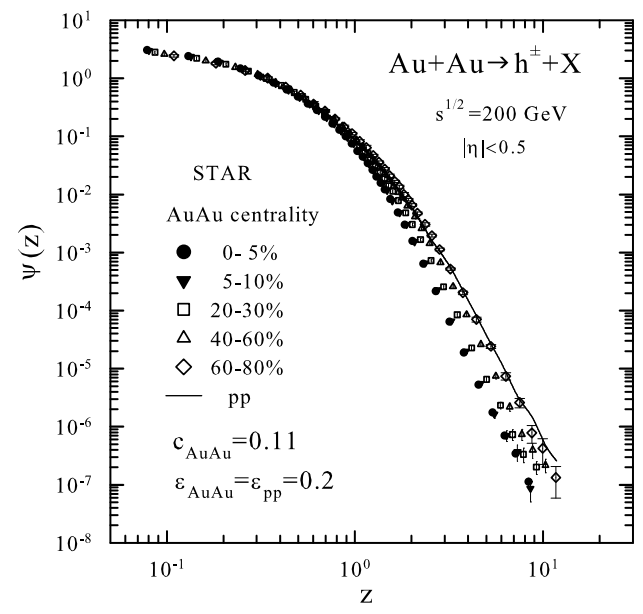

a)

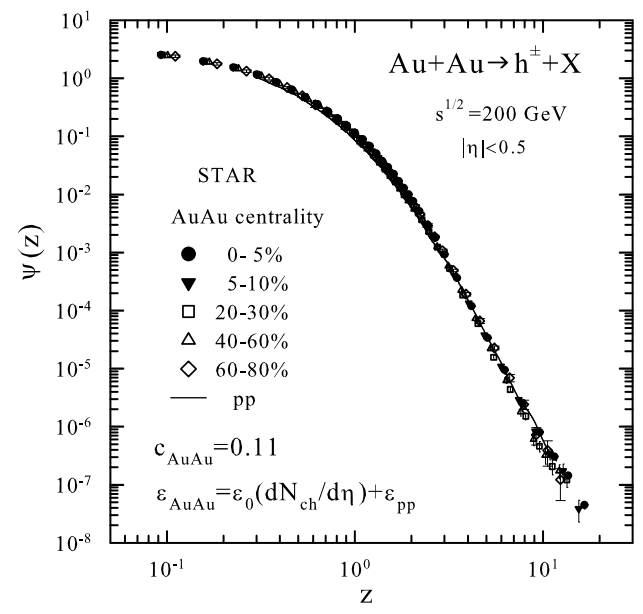

b)

Figure 9: Inclusive spectra of charged hadrons produced in $p p$ and $A u A u$ collisions for different centralities at $\sqrt{s_{N N}}=200 \mathrm{GeV}$ in $z$-presentation with (a) constant and (b) multiplicity dependent $\varepsilon$. Experimental data are taken from Ref. [5].

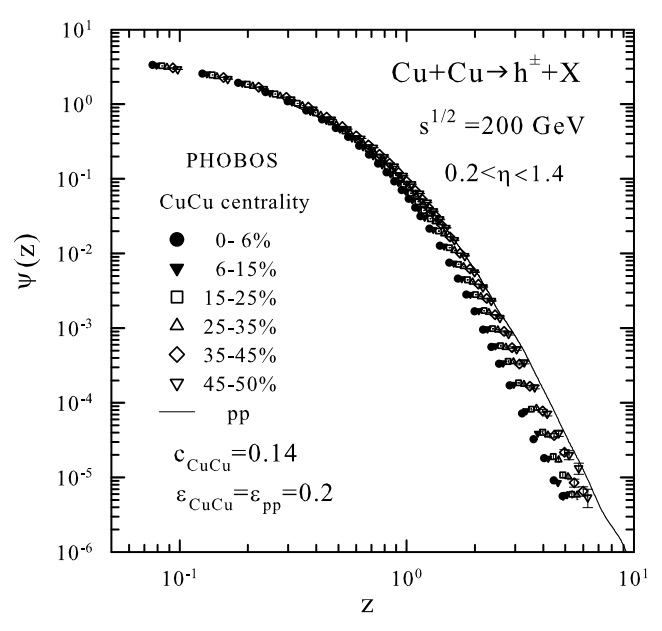

a)

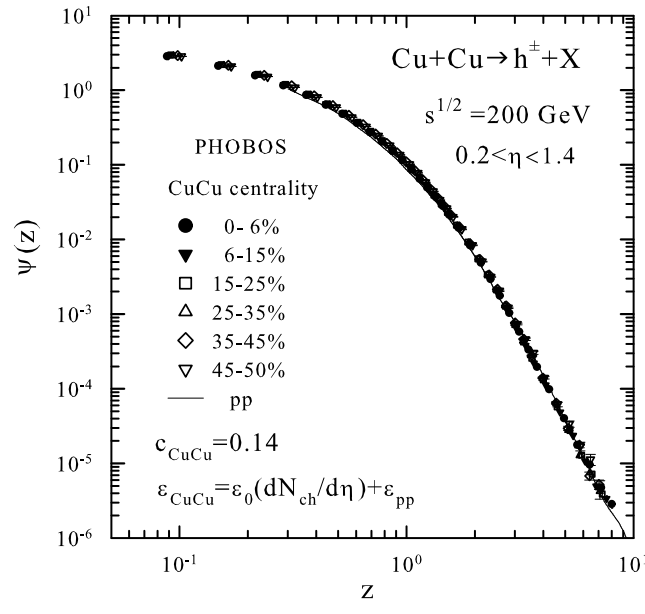

b)

Figure 10: Inclusive spectra of charged hadrons produced in $p p$ and $\mathrm{CuCu}$ collisions for different centralities at $\sqrt{s_{N N}}=200 \mathrm{GeV}$ in $z$-presentation with (a) constant and (b) multiplicity dependent parameter $\varepsilon$. Experimental data are taken from Refs. [5, 29].

is typically (10-20)\%, we observe a decrease of the "specific heat" with the increasing system size (A-dependence of specific heat). The suppression of the spectra in $\mathrm{CuCu}$ collisions relative to the $z$-scaling in proton-proton interactions is similar as for the $A u A u$ system. The deviation from the $p p$ curve enhances in the high- $z$ (high- $p_{T}$ ) region as the centrality of the $C u C u$ collisions increases. Figure 10 (b) demonstrates that multiplicity dependence of the parameter $\varepsilon_{\mathrm{CuCu}}$ in the form (4.1) with $\varepsilon_{0}=0.008$ allows us to restore the scaling shape of $\psi(z)$ for all multiplicity classes.

The demonstrated properties of the charged hadron spectra are typical also for pions. We illus- 
trate this on the STAR data [33] measured in $A u A u$ collisions at $\sqrt{s_{N N}}=200 \mathrm{GeV}$. The pion spectra

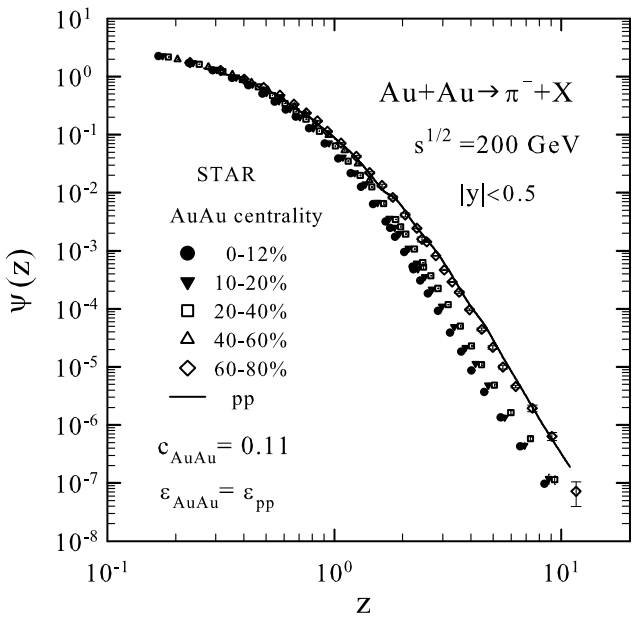

a)

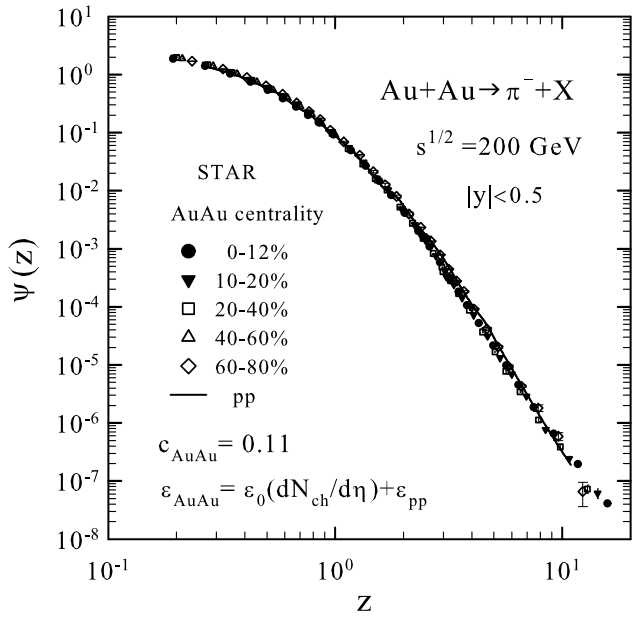

b)

Figure 11: The inclusive spectra of negative pions produced in $p p$ and $A u A u$ collisions [33] for different centralities at $\sqrt{s_{N N}}=200 \mathrm{GeV}$ in $z$-presentation for (a) constant and (b) multiplicity dependent parameter $\varepsilon$. The solid lines represent the scaling function for $p p$ collisions.

were taken at various centralities characterized by different multiplicity densities $d N_{\mathrm{ch}} /\left.d \eta\right|_{0}$ of charged particles produced in the central interaction region. The centrality dependence of $z$ presentation of the pion spectra is shown in Fig. 11(a). The parameter $\varepsilon \equiv \varepsilon_{\pi}=0.2$ was fixed at the same value as obtained from the scaling analysis of pions in the $p p$ collisions. The corresponding $p p$ data are represented by the solid line. The pion spectra in $p p$ and peripheral $A u A u$ collisions coincide each other with good accuracy in $z$-presentation for $c_{\mathrm{AuAu}}=0.11$. This value is consistent with energy independence of the scaling function for pions produced in the peripheral $A u A u$ interactions. As seen from Fig 11(a), $z$-presentation of the pion spectra for $A u A u$ collisions is suppressed in the high $z$ region relatively to the $z$-scaling as the centrality increases. The largest suppression corresponds to the most central collisions. Assuming the multiplicity dependence the parameter $\varepsilon$ in the form (4.1), the same scaling behavior as for $p p$ collisions can be obtained for $A u A u$ interactions for all centralities. This is illustrated in Fig.11(b) for the same value of the parameter $\varepsilon_{0}=0.0028$ as for charged particles.

\section{Energy losses in $p p \& A A$ collisions}

The approach based on the $z$-scaling concept allows us to develop a microscopic scenario of particle production in terms of the constituent interactions [10, 32]. Here we discuss some features of this scenario. The method of determination of the momentum fractions makes it possible to analyze kinematics of the constituent interactions in the framework of the developed approach. The dependence of the momentum fractions $y_{a}$ and $y_{b}$ on the kinematical variables $\left(p_{T}, \theta_{c m s}, \sqrt{s}\right)$ describes features of the fragmentation process. The fraction $y_{a}$ characterizes dissipation of the energy and momentum of particles produced by the underlying constituent interaction into the near side of the inclusive hadron. This effectively includes energy losses of the scattered partons moving 
in the direction of the registered hadron as well as feed down processes from prompt resonances out of which the inclusive particle may be created. The fraction $y_{b}$ governs the recoil mass $M_{X} \sim m_{2} / y_{b}$ in the constituent subprocess. Its value characterizes the dissipation of energy in the away side direction of the inclusive particle.

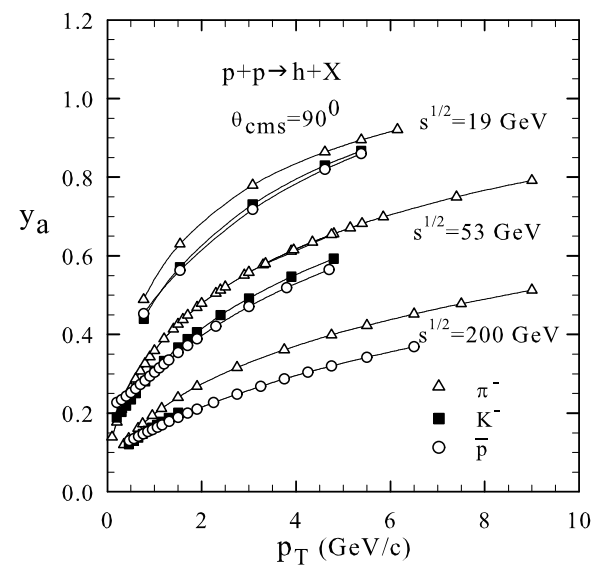

a)

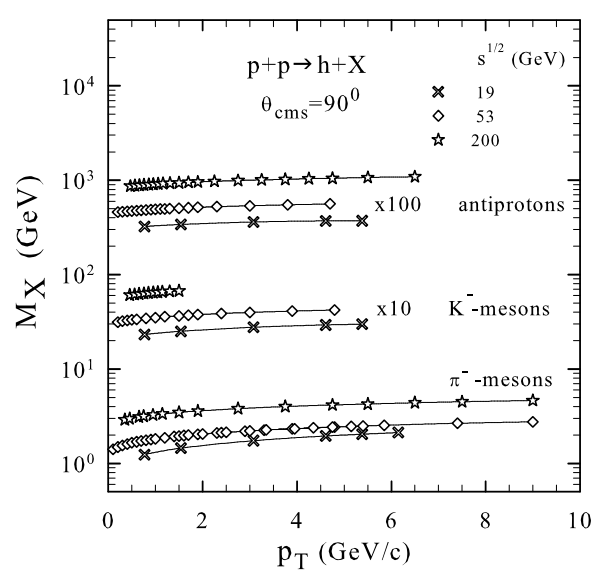

b)

Figure 12: The dependence of the fraction $y_{a}$ (a) and the recoil mass $M_{X}$ (b) on the transverse momentum $p_{T}$ for $\pi^{-}, K^{-}$, and $\bar{p}$ produced in the $p p$ collisions at $\sqrt{s}=19,53$, and $200 \mathrm{GeV}$. The symbols correspond to data measured at $\theta_{c m s}=90^{\circ}$.

Figure 12(a) shows the dependence of $y_{a}$ on the transverse momentum $p_{T}$ for the negative pions, kaons, and antiprotons produced in $p p$ collisions at the energies $\sqrt{s}=19,53,200 \mathrm{GeV}$ and $\theta_{c m s}=90^{\circ}$. All curves demonstrate a non-linear monotonic growth with $p_{T}$. It means that the energy dissipation associated with the production of a high- $p_{T}$ particle is smaller than for the inclusive processes with lower transverse momenta. This feature is similar for all inclusive reactions at all energies. The decrease of the fractions $y_{a}$ with the increasing collision energy is another property of the considered mechanism. It corresponds to more energy dissipation at higher energies. This can be due to the larger energy losses and/or due to the heavy prompt resonances. The third characteristic is a slight decrease of $y_{a}$ with the mass of the inclusive particle. It implies more energy dissipation for creation of heavier hadrons as compared with hadrons with smaller masses.

Figure 12(b) demonstrates the dependence of the recoil mass $M_{X}$ on the transverse momentum of the negative pions, kaons, and antiprotons produced in $p p$ collisions at the energies $\sqrt{s}=19,53$, and $200 \mathrm{GeV}$ in the central rapidity region. For the sake of clarity, the values of $M_{X}$ are presented on a $\log$-scale with the multiplication factors 10 and 100 for $K^{-}$and $\bar{p}$, respectively. All curves demonstrate small growth at low $p_{T}$ followed by a successive flattening. They reveal a characteristic increase with the collision energy and mass of the inclusive particle. The qualitative properties of the $p_{T}$ dependence of $M_{X}$ are similar for different hadrons. The corresponding values of the momentum fraction $y_{b}$ are much less than $y_{a}$ for $p_{T}>1 \mathrm{GeV} / \mathrm{c}$. This means that, for sufficiently large $p_{T}$, the momentum balance in the production of an inclusive particle from a subprocess is more likely compensated with many particles with smaller momenta than by a single particle with a higher momentum moving in the opposite direction. 


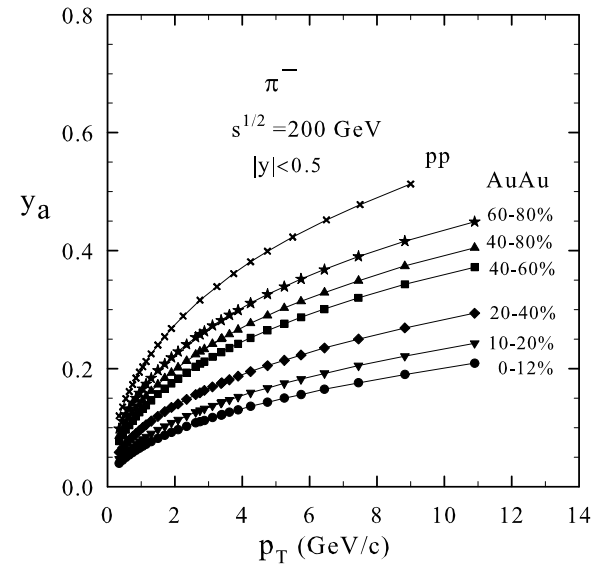

a)

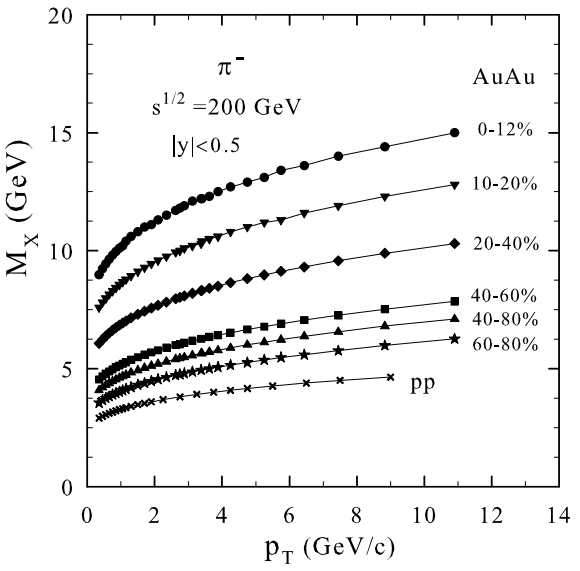

b)

Figure 13: The dependence of the fraction $y_{a}$ (a) and the recoil mass $M_{X}$ (b) on the transverse momentum $p_{T}$ for $\pi^{-}$-mesons produced in $p p$ and $A u A u$ collisions at different centralities. The symbols correspond to data measured at $\sqrt{s_{N N}}=200 \mathrm{GeV}$ and $|y|<0.5$.

The dependencies of the fraction $y_{a}$ and the recoil mass $M_{X}$ on the transverse momentum $p_{T}$ of $\pi^{-}$-mesons produced in $p p$ and $A u A u$ collisions at $\sqrt{s_{N N}}=200 \mathrm{GeV}$ are shown in Figs. 13(a) and 13(b), respectively. The decrease of $y_{a}$ with centrality in $A u A u$ collisions represents larger energy losses in the central collisions as compared with the $p p$ and peripheral $A u A u$ interactions. A slight increase of the recoil mass $M_{X}$ with $p_{T}$ is characteristic for $p p$ collisions. The values of $M_{X}$ and their growth with $p_{T}$ become larger with centrality in $A u A u$ interactions. This means that the momentum balance in a subprocess underlying the pion production is compensated with growing number of particles moving in the away side direction when the centrality of the nuclear collisions increases.

\section{QCD test of $z$-scaling for jets}

In this section we present a comparison of the $z$-scaling analysis for jet production in $\bar{p} p$ collisions with calculations performed in the framework of QCD. The code from Ellis-Kunszt-Soper [34] was used for calculations of inclusive cross sections in the next-to-leading order (NLO) of QCD. The NLO QCD calculations by EKS group are based on the matrix elements published in Refs. [35, 36]. The parton distribution functions CTEQ6M [37] and MRST01 [38] have been used in our calculations. They incorporate a large amount of experimental information evaluated at the NLO level. The NLO QCD calculations are influenced by the factorization $\left(\mu_{F}\right)$ and renormalization $\left(\mu_{R}\right)$ scales. Typical values of these parameters vary in the range $\left(p_{T} / 2,2 p_{T}\right)$. The values of the renormalization and factorization scales were taken to be $\mu_{R}=\mu_{F}=p_{T} / 2$. The calculation results depend also on the jet-cone radius $R=\left[(\Delta \eta)^{2}+(\Delta \phi)^{2}\right]^{1 / 2}$ in the pseudorapidity and azimuth $\{\eta, \phi\}$ space and the jet-separation parameter $R_{\text {sep }}$. The relation $R_{\text {sep }}=2 R$ between both parameters and the "optimal value" $R=0.7$ of the cone radius was used. The $\overline{\mathrm{MS}}$ scheme was exploited to subtract final state collinear singularities. The strong coupling $\alpha_{S}\left(\mu_{R}\right)$ was defined in the $\overline{\mathrm{MS}}$ renormalization scheme at the scale $\mu_{R}$. 
The invariant cross sections of jet production as a function of the collision energy $\sqrt{s}$ and transverse momentum $p_{T}$ calculated in the NLO QCD are shown in Fig. 14. The spectra were calculated over a wide range of the transverse momentum $p_{T}=10-3000 \mathrm{GeV} / c$ and the energy $\sqrt{s}=63-14000 \mathrm{GeV}$ at $\theta_{c m s} \simeq 90^{\circ}$. As seen from Fig. 14 the strong dependence of the spectra on the collision energy increases with $p_{T}$. The experimental data [22,23] on jet cross sections at $\sqrt{s}=630,1800$, and $1960 \mathrm{GeV}$ obtained by the D0 Collaboration are shown by the symbols. The same data are plotted in the dependence on the variable $z$ in Fig. 15. The solid line represents the asymptotic behavior of the scaling function $\psi(z)$ obtained from analysis of the available data. The value of the slope parameter was found to be $\beta=5.48 \pm 0.02$. The $z$-presentation of the NLO QCD calculations at different energies is shown by the dashed lines.

A good agreement between the experimental data and the corresponding NLO QCD calculations is observed. One can see, however, that the NLO QCD predictions demonstrate considerable deviation from the asymptotic behavior of $\psi(z)$ predicted by the $z$-scaling as the collision energy and transverse momentum increase. The deviation is clearly manifested in the region where the experimental measurements are not performed yet. There are some factors which can modify the asymptotic behavior of $\psi(z)$. They are quark and gluon distribution functions (PDF) and the fragmentation function (FF) of a quark or gluon into the jet. The latter is a rather complex process which should be taken into account in the QCD calculations as well. The large uncertainties in the gluon distribution function may be essentially restricted by the power behavior of $\psi(z)$ established over a wide range of $\sqrt{s}$ and high $p_{T}$. This behavior could give new additional constraint on PDFs and FFs which are phenomenological quantities weakly controlled by the perturbative QCD.

On the basis of the obtained results we conclude that the self-similar features of experimental data on jet production in $\bar{p} p$ collisions give strong restriction on the asymptotic behavior of the scaling function $\psi(z)$. The behavior is not reproduced by the NLO QCD evolution of cross sections with the phenomenological parton distribution functions used in the present analysis in the region

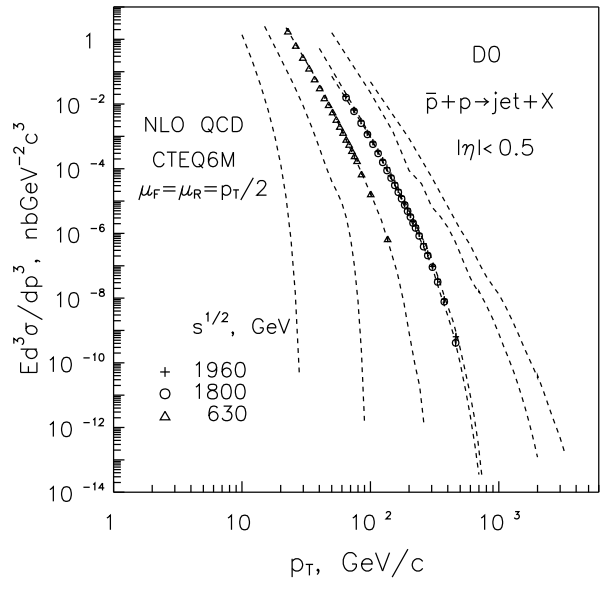

a)

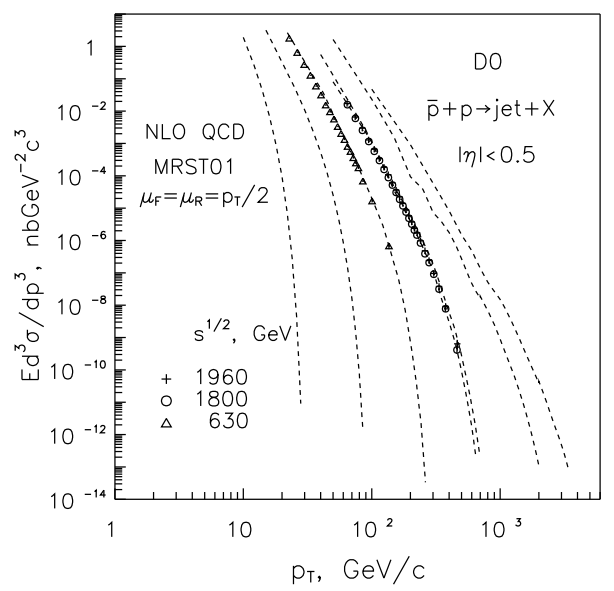

b)

Figure 14: The dependence of the inclusive cross sections of jet production in $\bar{p} p$ collisions on the transverse momentum $p_{T}$. Dashed lines are calculated results in NLO QCD with (a) CTEQ6M [37] and (b) MRST01 [38] PDFs at $\sqrt{s}=63,200,630,1800,1960,7000$, and $14000 \mathrm{GeV}$. Points are the experimental data [22, 23] obtained by the D0 Collaboration. 


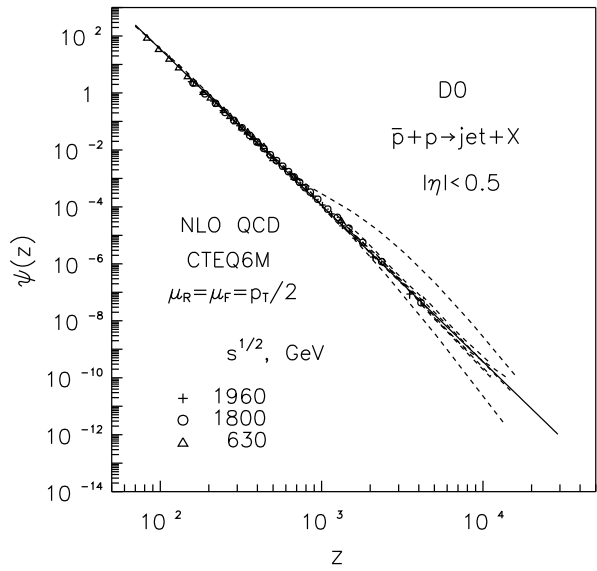

b)

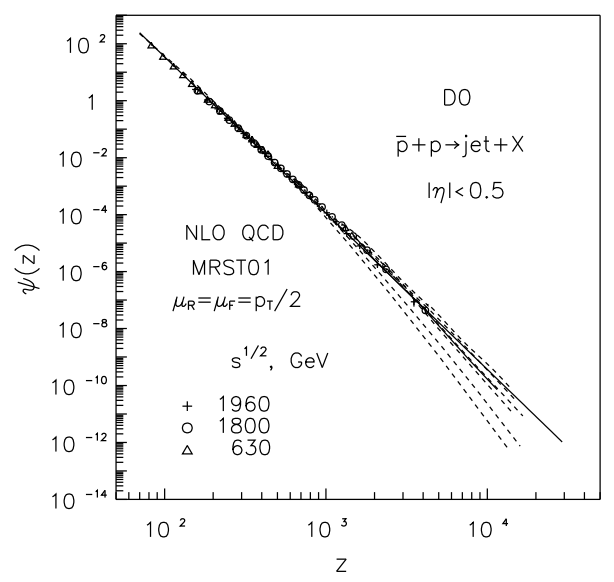

c)

Figure 15: The scaling function $\psi(z)$ of jet production in $\bar{p} p$ collisions versus $z$. The dashed lines are calculated results in the NLO QCD with (a) CTEQ6M [37] and (b) MRST01 [38] PDFs at $\sqrt{s}=$ $63,200,630,1800,1960,7000$, and $14000 \mathrm{GeV}$. The points $(\triangle, \circ,+)$ represent the experimental data $[22,23]$ at $\sqrt{s}=630,1800$, and $1960 \mathrm{GeV}$.

where experimental measurements are expected in the future.

\section{Conclusions}

We have studied the spectra of the identified hadrons produced in $p p$ and $\bar{p} p$ collisions in $z$ presentation. Flavor independence of the scaling function $\psi(z)$ was demonstrated. This includes hadrons with light and heavy quarks produced over a wide region of the transverse momenta and production angles. A saturation regime of $\psi(z)$ was observed for $z<0.1$. Transverse momentum distributions of the direct photons and jets obtained at the Tevatron were analyzed in framework of the $z$-scaling. These data confirm the energy and angular independence of the scaling function $\psi(z)$. The power behavior, $\psi(z) \sim z^{-\beta}$, is observed up to the highest measured transverse momentum $p_{T} \simeq 600 \mathrm{GeV} / \mathrm{c}$. The obtained results manifest self-similarity, locality, and fractality of the direct photon and jet production in hadron interactions at a constituent level.

We have analyzed experimental data on charged hadron spectra in $A u A u$ and $C u C u$ collisions at the RHIC in terms of the variable $z$. The data in $z$-presentation indicate similarity as a characteristic feature of mechanism of hadron production. This property includes structure of the colliding objects, interaction of their constituents, and character of the fragmentation process. We argue that self-similarity is a general property of the soft and hard hadron interactions at high energies. The analysis of spectra of charged hadrons and pions shows that the parameter $c$ interpreted as a "specific heat" of the medium produced in heavy ion collisions is independent of the collision energy and centrality. Its value decreases with the increasing system size. A dependence of the fragmentation dimension $\varepsilon_{A A}$ on the collision centrality allows us to restore the unique shape of the scaling function for $p p$ and $A A$ collisions for charged hadrons and pions over a wide range of $z$. The scaling function manifests typical power behavior, $\psi(z) \simeq z^{-\beta}$, in the hard $p_{T}$ region (large $z$ ) with a constant value of the slope parameter $\beta$. 
A microscopic scenario of proton-proton and nucleus-nucleus interactions at a constituent level in terms of momentum fractions was developed. It allows to estimate the energy losses of the secondary partons passing through the medium created in the nuclear interactions in dependence on the collision centrality. It was shown that energy losses increase in a specific manner with the collision energy and centrality, and decrease with $p_{T}$.

A test of the $z$-scaling for jet production in $\bar{p} p$ collisions was performed in the framework of QCD. The inclusive cross sections were calculated in NLO approximation with the CTEQ6M and MRST01 parton distribution functions. The results of calculations were compared with the scaling function $\psi(z)$ obtained from analysis of existing experimental data. It was shown that selfsimilar features of jet production dictated by the $z$-scaling give strong restriction on the asymptotic behavior of $\psi(z)$ and thus on inclusive spectra in high- $p_{T}$ region. The restriction could be used as a constraint on gluon distribution functions in a global QCD analysis of experimental data.

Verification of the hypothesis on universality of the asymptotic behavior of the scaling function and more precise study of the $z$-scaling in hadron, direct photon, and jet production is possible in $\bar{p} p$ and $p p$ collisions at Tevatron, RHIC, and LHC. A violation of the $z$-scaling is suggested as a signature of new phenomena at high energies.

\section{Acknowledgments}

The investigations have been supported by the IRP AVOZ10480505, by the Grant Agency of the Czech Republic under Contract No. 202/07/0079 and by the special program of the Ministry of Science and Education of the Russian Federation, grant RNP.2.1.1.2512.

\section{References}

[1] R. P. Feynman, Phys. Rev. Lett. 23, 1415 (1969).

[2] A.M. Polyakov, Zh. Eksp. Theor. Fiz. 59, 542 (1970); Zh. Eksp. Theor. Fiz. 60, 1572 (1971).

[3] Z. Koba, H. B. Nielsen, and P. Olesen, Nucl. Phys. B 40, 317 (1972).

[4] J. Cronin et al., Phys. Rev. D 11, 3105 (1975).

[5] J. Adams et al. [STAR Collaboration], Phys. Rev. Lett. 91, 172302 (2003).

[6] I. Arsene et al. [BRAHMS Collaboration], Phys. Rev. Lett. 91, 172305 (2003).

[7] S. S. Adler et al. [PHENIX Collaboration], Phys. Rev. C 69, 034910 (2004).

[8] B. B. Back et al. [PHOBOS Collaboration], Phys. Rev. Lett. 94, 082304 (2005).

[9] I. Zborovský and M.V. Tokarev, Phys. Rev. D 75, 094008 (2007).

[10] I. Zborovský and M.V. Tokarev, Int. J. Mod. Phys. A 24, 1417 (2009).

[11] M.V. Tokarev and I. Zborovský, Yad. Fiz. 70, 1335 (2007).

[12] V. S. Stavinsky, Sov. J. Part. Nucl. 10, 949 (1979).

[13] I. Zborovský, M.V. Tokarev, Yu.A. Panebratsev, and G.P. Škoro, Phys. Rev. C 59, 2227 (1999). 
[14] D. Antreasyan et al., Phys. Rev. D 19, 764 (1979).

B. Alper et al. [BS Collaboration], Nucl. Phys. B 100, 237 (1975).

F. W. Büsser et al. [CCRS Collaboration], Nucl. Phys. B 106, 1 (1976).

D.E. Jaffe et al., Phys. Rev. D 40, 2777 (1989).

J. Adams et al. [STAR Collaboration], Phys. Lett. B 637, 161 (2006).

J. Adams et al. [STAR Collaboration], Phys. Lett. B 616, 8 (2005).

[15] B.I. Abelev et al. [STAR Collaboration], Phys. Rev. C 75, 064901 (2007).

[16] M.G. Albrow et al. [CHLM Collaboration], Nucl. Phys. B 56, 333 (1973).

[17] K. Guettler et al. [BSM Collaboration], Phys. Lett. B 64, 111 (1976).

K. Guettler et al. [BSM Collaboration], Nucl. Phys. B 116, 77 (1976).

[18] J. Adams et al. [STAR Collaboration], Phys. Rev. Lett. 92, 092301 (2004).

S.S. Adler et al. [PHENIX Collaboration], Phys. Rev. C 75, 051902(R) (2007).

J. Adams et al. [STAR Collaboration], Phys. Lett. B 612, 181 (2005).

J. Adams et al. [STAR Collaboration], Phys. Rev. C 71, 064902 (2005).

[19] D. Acosta et al. [CDF Collaboration], Phys. Rev. D 71, 032001 (2005).

D. Acosta et al. [CDF Collaboration], Phys. Rev. Lett. 88, 161802 (2002).

D. Acosta et al. [CDF II Collaboration], Phys. Rev. Lett. 91, 241804 (2003).

[20] B. Abbott et al. [D0 Collaboration], Phys. Rev.Lett. 84, 2786 (2000).

V.M. Abazov et al. [D0 Collaboration], Phys. Lett. B 639, 151 (2006).

[21] M. Tokarev, G. Efimov, arXiv:hep-ph/0209013.

M.V. Tokarev, G.L. Efimov, and D.E. Toivonen, Phys. At. Nucl. 67, 564 (2004).

[22] B. Abbott et al. [D0 Collaboration], Phys. Rev. Lett. 82, 2451 (1999).

B. Abbott et al. [D0 Collaboration], Phys. Rev. D 64, 032003 (2001).

D. Elvira, Ph.D Thesis Universodad de Buenos Aires, Argentina (1995).

V.M. Abazov et al. [D0 Collaboration], Phys. Lett. B 525, (2002) 211 [arXiv:hep-ex/0109041], [arXiv:hep-ex/0106032].

[23] M. Begel et al. [D0 Collaboration], arXiv:hep-ex/0305072.

M. Voutilainen (for D0 Collaboration) XIV Interbational Workshops on Deep-Inelastic Scattering and Related Subjects (DIS2006), April 20-24, 2006, Tsukuba, Japan; http://www-conf.kek.jp/dis06/ J. Commin (for D0 Collaboration) XV Interbational Workshops on Deep-Inelastic Scattering and Related Subjects (DIS2007), April 16-20, 2007, Munich, Germany; http://www.mppmu.mpg.de/dis2007/

[24] F. Abe et al. [CDF Collaboration], Phys. Rev. Lett. 77, 438 (1996).

T. Affolder et al. [CDF Collaboration], Phys. Rev. D 64, 032001 (2001).

[25] A. Abulencia et al. [CDF Collaboration], Phys. Rev. Lett. 96, 122001 (2006) [arXiv:hep-ex/0512062].

A. Abulencia et al. [CDF Collaboration], Phys. Rev. D 74, 071103 (2006) [arXiv:hep-ex/0512020].

[26] A. Abulencia et al. [CDF Collaboration], Phys. Rev. D 75, 092006 (2007).

[27] V.M. Abazov et al. [D0 Collaboration], Phys. Rev. Lett. 101, 062001 (2008).

[28] C. Adler et al. [STAR Collaboration], Phys. Rev. Lett. 89, 202301 (2002).

[29] B. Alver et al. [PHOBOS Collaboration], Phys. Rev. Lett. 96, 212301 (2006).

[30] A. Breakstone et al. [ABCDHW Collaboration], Z. Phys. C 69, 55 (1995). 
[31] D. Drijard et al. [CDHW Collaboration], Nucl. Phys. B 208, 1 (1982).

[32] M.V. Tokarev and I. Zborovský, Yad. Fiz. 72, 588 (2009) [Phys. At. Nucl. 72, 552 (2009)].

[33] B.I. Abelev et al. [STAR Collaboration], Phys. Rev. Lett. 97, 152301 (2006).

[34] S.D. Ellis, Z. Kunszt, and D. Soper, Phys. Rev. Lett. 69, 1496 (1992); http://zebu.uoregon.edu/ ${ }^{\sim}$ soper/soper.html

[35] S.D. Ellis, Z. Kunszt, and D. Soper, Phys. Rev. D 40, 2188 (1989).

[36] Z. Kunszt, D. Soper, Phys. Rev. D 46, 192 (1992).

[37] J. Pumplin et al., JHEP 0207, 012 (2002); [arXiv:hep-ph/0201195]; http://www.phys.psu.edu/ $\sim$ cteq

[38] A.D. Martin et al., Eur. Phys. J. C 23, 73 (2002); [arXiv:hep-ph/0110215]; http://www.durpdg.dur.ac.uk/hepdata/mrs 